\title{
Application of Compound Preservatives in Beijing Sausages
}

\author{
Gao Xiang ${ }^{1,2}$, Han Mengmeng ${ }^{1,2}$, Cai Ziyi ${ }^{1,2}$, Zhang Xingyuan ${ }^{1,2}$, Wang Fang ${ }^{1,2,3}$, Pang Meixia ${ }^{1,2,3}$, \\ Qi Jinghua ${ }^{1,2,3, *}$
}

${ }^{1}$ Beijing Key Laboratory of Agricultural Product Detection and Control of Spoil age Organisms and Pesticide Residue, Beijing University of Agriculture, Beijing, China

${ }^{2}$ The Teaching Group of Food Chemistry, Faculty of Food Science and Engineering, Beijing University of Agriculture, Beijing, China

${ }^{3}$ Beijing Innovation Consortium of Swine Research System, Beijing, China

\section{Email address:}

17835423121@163.com(Gao Xiang), abc960718@sina.com(Qi Jinghua)

${ }^{*}$ Corresponding author

\section{To cite this article:}

Gao Xiang, Han Mengmeng, Cai Ziyi, Zhang Xingyuan, Wang Fang, Pang Meixia, Qi Jinghua. Application of Compound Preservatives in Beijing Sausages. American Journal of Bioscience and Bioengineering. Vol. 7, No. 2, 2019, pp. 46-50. doi: 10.11648/j.bio.20190702.14

Received: April 15, 2019; Accepted: May 26, 2019; Published: June 15, 2019

\begin{abstract}
The purpose of this study was to explore the effect of natural and safe compound preservatives on the shelf life of Beijing sausages. In this thesis, Pseudomonas sp was the first dominant bacterium in the Beijing sausages, which was taken as the research object. The total number of bacteria and the sensory score were taken as the evaluation indexes. On the basis of previous studies, Cinnamon, clove and fennel had good antibacterial effect and those were selected as compound preservatives. By using three-factor quadratic universal rotation design experiment and establishing the mathematical model. Then the effect of single factor experiment shown that within the coding range, the bacteriostatic effects of anise, cinnamon and clove were gradually weakened, the effect of interaction experiment shown that there was significant interaction between cinnamon and clove. When the total number of bacteria reached the lowest level, the optimal ratio of the compound preservatives was determined as follows: cinnamon $0.063 \mathrm{~g} / \mathrm{mL}$, clove $1.000 \mathrm{~g} / \mathrm{mL}$, fennel $0.810 \mathrm{~g} / \mathrm{mL}$. According to this formula, $0.1 \%$ of compound preservative was added to the meat and compared to the control group, under the same conditions and time of storage, the total number of bacteria was significantly lower. The result shown that The shelf life of sausages could be extended by 9 days by adding $0.1 \%$ compound preservatives.
\end{abstract}

Keywords: Compound Preservatives, Beijing Sausages, Application

\section{Introduction}

The Beijing sausage is a kind of low-temperature meat products, which has high quality, delicious taste and strong fragrance. However, in the process of storage, transportation and marketing, due to the increase of microorganisms, it is easy to spoil and decay, and the shelf life of product is often short, which greatly limits the development of such products [1]. Natural spices, as common food condiments and preservatives, are healthy, non-toxic and safe [2-3]. Spices such as anise, clove and cinnamon are common excipients in meat processing [4-6]. They have bacteriostatic, antioxidant and flavoring effects [7-11]. Hernndez found that the inhibitory concentration of cumin and clove volatile oil on five common pathogenic bacteria in meat was $500 \mathrm{mg} / \mathrm{L}$ and $750 \mathrm{mg} / \mathrm{L}$, respectively, which had significant effect on meat preservation [12]. Cinnamon extract has obvious bacteriostatic effect on Pseudomonas spp., Cyclosporus thermophilus and Escherichia coli [13]. Benmalek and others extracted flavonols and anthocyanins from fennel. The results show that these substances have good inhibitory effect on Escherichia coli. Their water-soluble volatile components had broad-spectrum antimicrobial activity, and their antimicrobial activity was obviously stronger than the oil phase of fennel volatile components [14-15]. Tian Feng found that anise, cinnamon and clove showed obvious bacteriostatic effect [16]. Zhu Jiankai's beef prepared with natural spices could be stored for more than 18 days, 3-6 
days longer than the general storage [17]. The purpose of this article is to explore a natural compound preservative to prolong the shelf life of Beijing sausages.

\section{Materials}

\subsection{Materials}

Natural spices, purchased at Beijing Huilong Guanjing Huitang Peking Pharmacy, meat, purchased at Qiliqu Agricultural and Trade Market

\subsection{Sample Preparation}

The natural spices were removed from impurities. After crushing by crusher, they were sieved by 40 meshes and soaked in solvent for 12 hours. The extraction was carried out by ultrasonic-assisted extraction for 1 hour. Then, the solution was purified and volume-fixed into a volumetric bottle (concentration $1 \mathrm{~g} / \mathrm{mL}$ ), and stored in a refrigerator at $4^{\circ} \mathrm{C}$ for reserve.

The meat was processed by the Beijing sausages processing technology of PengCheng Food Company.

\section{Methods}

\subsection{Activation of Indicator Bacteria}

Pseudomonas sp. frozen with glycerol was restored at normal temperature. It was poured into $50 \mathrm{~mL}$ sterilized nutritious broth medium and cultured at $30^{\circ} \mathrm{C}$ for 12 hours.

\subsection{Three-Factor Quadratic General Rotary Test Design}

Using three-factor quadratic general rotary design test [18, 19], the selected three preservatives cinnamon, clove and anise were divided into five levels (the highest extracting concentration of spices was $1 \mathrm{~g} / \mathrm{mL}$ and the lowest bacteriostatic concentration was the lowest). Cinnamon, clove and anise were expressed by $X_{1}, X_{2}$ and $X_{3}$, respectively. According to the level coded in table 1 , the concentrations of cinnamon, clove and anise were the highest. Aseptic distilled water was used to prepare the solution.

Table 1. Codes of the factor levels.

\begin{tabular}{llll}
\hline Coding value & $\begin{array}{l}\text { Cinnamon } \\
\mathbf{X}_{\mathbf{1}}(\mathbf{g} / \mathbf{m L})\end{array}$ & $\begin{array}{l}\text { Clove } \\
\mathbf{X}_{\mathbf{2}}(\mathbf{g} / \mathbf{m L})\end{array}$ & $\begin{array}{l}\text { Star anise } \\
\mathbf{X}_{\mathbf{3}}(\mathbf{g} / \mathbf{m L})\end{array}$ \\
\hline Upper arm number +1.682 & 1.000 & 1.000 & 1.000 \\
Upper level +1 & 0.810 & 0.800 & 0.810 \\
Benchmark level 0 & 0.532 & 0.508 & 0.532 \\
Lower level -1 & 0.253 & 0.215 & 0.253 \\
Lower arm number -1.682 & 0.063 & 0.016 & 0.063 \\
\hline
\end{tabular}

By doing bacteriostatic experiments. The total bacterial count $(\mathrm{CFU} / \mathrm{mL}) / 10^{5}$ was taken as the evaluation index value $\mathrm{Y}$. According to the three-factor quadratic general rotary design part of DPS agricultural experiment statistical analysis software, the evaluation value $\mathrm{Y}$ was input into the computer to get the statistical results. The results were analyzed by multiple regression analysis using the analysis software, and the regression equation was obtained for variance analysis.

\subsection{Effect of Compound Preservatives on Beijing Sausages}

After obtaining the formula of compound preservative, added it to the sausages and carried out sensory evaluation test on the samples on the first day of production. During the storage period of the samples, the microbial indexes of the samples were analyzed to study the antiseptic effect of composite preservatives on the Beijing sausages.

\section{Results and Discussion}

\subsection{Test Results of Quadratic General Rotary Design with Three Factors}

Table 2. Results of three factors quadratic rotation design.

\begin{tabular}{lllll}
\hline Test number & $\mathbf{X}_{\mathbf{1}}$ & $\mathbf{X}_{\mathbf{2}}$ & $\mathbf{X}_{\mathbf{3}}$ & $\mathbf{Y}$ \\
\hline 1 & 1 & 1 & 1 & 21 \\
2 & 1 & 1 & -1 & 28 \\
3 & 1 & -1 & 1 & 32 \\
4 & 1 & -1 & -1 & 41 \\
5 & -1 & 1 & 1 & 30 \\
6 & -1 & 1 & -1 & 49 \\
7 & -1 & -1 & 1 & 84 \\
8 & -1 & -1 & -1 & 137 \\
9 & 1.682 & 0 & 0 & 30 \\
10 & -1.682 & 0 & 0 & 79 \\
11 & 0 & 1.682 & 0 & 24 \\
12 & 0 & -1.682 & 0 & 93 \\
13 & 0 & 0 & 1.682 & 69 \\
14 & 0 & 0 & -1.682 & 116 \\
15 & 0 & 0 & 0 & 52 \\
16 & 0 & 0 & 0 & 56 \\
17 & 0 & 0 & 0 & 62 \\
18 & 0 & 0 & 0 & 49 \\
19 & 0 & 0 & 0 & 44 \\
20 & 0 & 0 & 0 & 59 \\
\hline
\end{tabular}

\subsection{Variance Analysis}

The data in Table 2 were analyzed by the statistical analysis software of DPS agricultural experiment. The regression equation was obtained with the total number of bacteria $(\mathrm{CFU} / \mathrm{mL}) / 10^{5}$ as dependent variable and the additive concentration of cinnamon, clove and anise as independent variables.

$$
\begin{aligned}
& Y=54.20199-19.06672 X 1-20.65111 X 2-12.23100 \times 3-3.21673 X 12 \\
& -1.80286 X 22+10.21499 X 32+14.75000 X 1 X 2+7.00000 X 1 X 3+4.50000 X 2 X 3
\end{aligned}
$$

Variance analysis of the variables in the regression equation is carried out, and the results are shown in Table 3. 
Table 3. Result of variance analysis.

\begin{tabular}{|c|c|c|c|c|c|c|}
\hline Source of variation & Sum of squares & Freedom & mean square & partial correlation & F value & P value \\
\hline $\mathrm{X}_{1}$ & 4965.3173 & 1 & 4965.3173 & 0.8791 & 34.0164 & 0.0002 \\
\hline $\mathrm{X}_{2}$ & 5824.8112 & 1 & 5824.8112 & 0.8942 & 39.9046 & 0.0001 \\
\hline $\mathrm{X}_{3}$ & 2043.2371 & 1 & 2043.2371 & 0.7637 & 13.9978 & 0.0038 \\
\hline $\mathrm{X}_{1}^{2}$ & 149.1733 & 1 & 149.1733 & 0.3045 & 1.022 & 0.3359 \\
\hline $\mathrm{X}_{2}^{2}$ & 46.8584 & 1 & 46.8584 & 0.1764 & 0.321 & 0.5835 \\
\hline $\mathrm{X}_{3}^{2}$ & 1504.3153 & 1 & 1504.3153 & -0.7124 & 10.3058 & 0.0093 \\
\hline $\mathrm{X}_{1} \mathrm{X}_{2}$ & 1740.5 & 1 & 1740.5 & -0.7375 & 11.9238 & 0.0062 \\
\hline $\mathrm{X}_{1} \mathrm{X}_{3}$ & 392 & 1 & 392 & -0.4601 & 2.6855 & 0.1323 \\
\hline $\mathrm{X}_{2} \mathrm{X}_{3}$ & 162 & 1 & 162 & -0.3161 & 1.1098 & 0.3169 \\
\hline regression & 16980.0659 & 9 & 1886.674 & $\mathrm{~F} 2=12.92522$ & & 0.0002 \\
\hline Surplus & 1459.6841 & 10 & 145.9684 & & & \\
\hline Loss of imitation & 1238.3508 & 5 & 247.6702 & $F 1=5.59496$ & & 0.051 \\
\hline deviation & 221.3333 & 5 & 44.2667 & & & \\
\hline sum & 18439.75 & 19 & & & & \\
\hline
\end{tabular}

The result of variance analysis were shown that the $p$ value of $\mathrm{F}$ test corresponding to the regression equation was 0.0002 $<0.05$, which reached a very significant level. It showed that the regression model was effective and could reflect the actual situation in the experiment to the maximum extent. The $p$ value of the missing item was equal to $0.051>0.05$, which showed that the model fit the test well and the test error was small.

And the effects of $X_{1}, X_{2}$ and $X_{3}$ on $Y$ value were highly significant, and the absolute values of regression coefficient of quadratic terms of regression equation were $\mathrm{X}_{3}>\mathrm{X}_{1}>\mathrm{X}_{2}$ in turn, indicating that among the compound preservatives, anise had the most significant bacteriostatic effect on Pseudomonas, followed by cinnamon and clove. In addition, there was a significant interaction between cinnamon and clove, but there was no significant interaction between cinnamon and anise, clove and anise.

\subsection{Analysis Results of Single Factor Effects}

Two factors in the regression equation were controlled at zero level, and the single factor effect equation was obtained to analyze the bacteriostatic effect of a single spice on Pseudomonas sp. The three single-factor effect equations are as follows:

$$
\begin{gathered}
\mathrm{Y} 1=54.20199-19.06672 \times 1-3.21673 \times 12 \\
Y 2=54.20199-20.65111 \times 2-1.80286 \times 22 \\
Y 3=54.20199-12.23100 \times 3+10.21499 \times 32
\end{gathered}
$$

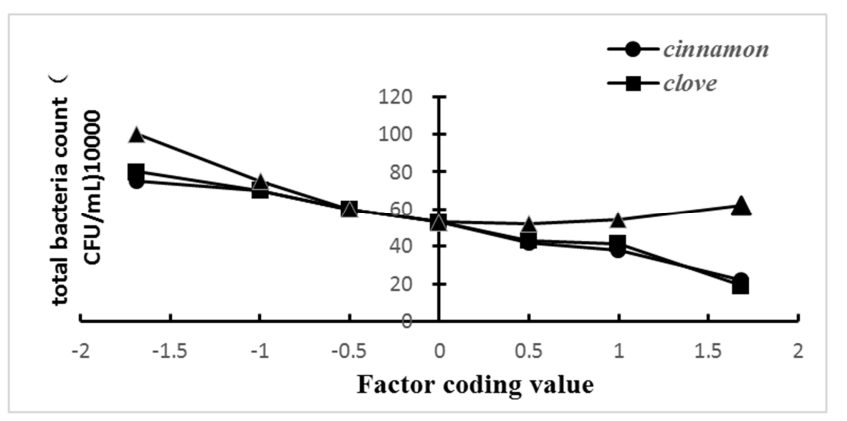

Figure 1. The curve of single factor effect.
According to the single factor equation, the single factor effect curve is obtained, as shown in Figure 2. It showed that the bacteriostatic effect increases with the increase of cinnamon and clove dosage, and the bacteriostatic effect of anise increased first and then decreased with the increase of its concentration.

\subsection{Marginal Effect Analysis}

The first-order partial derivative of the single-factor effect equation is obtained, and the single-factor marginal effect equation is obtained.

$$
\begin{aligned}
& \text { d Y1/d X1=-19.06672-6.43346X1 } \\
& \text { d Y2/d X2=-20.65111-3.60572X2 } \\
& \text { d Y3/d X3=-12.23100+20.42998X3 }
\end{aligned}
$$

The single factor marginal effect equation reflected the change rate of $\mathrm{Y}$ value (total number of Pseudomonas colonies) with three different spice coding values (concentration level). According to the single factor marginal effect equation, the single factor marginal effect map is obtained, as shown in Figure 2.

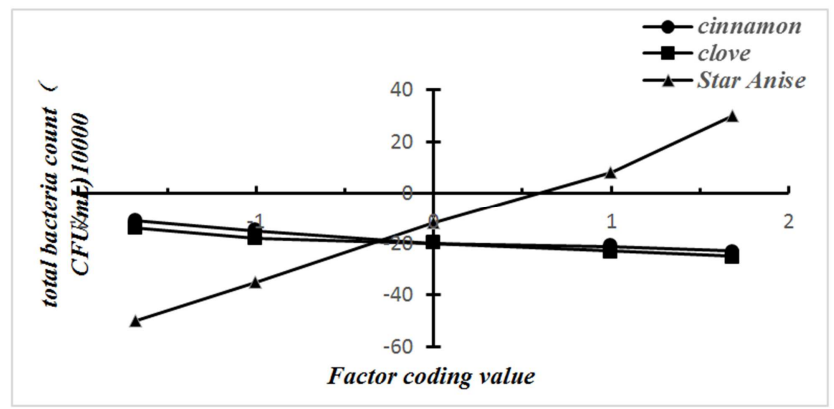

Figure 2. The curve of single factor boundary effect.

It showed that the concentration of anise had the greatest influence on the total bacterial count, followed by cinnamon and clove. In addition, at higher concentrations, the anise concentration on the change rate of total bacterial count was increasing. 


\subsection{Interaction Effect Analysis Results}

The regression equation was analyzed by dimensionality reduction. Another factor (anise) was fixed at zero level, and the interaction equation between cinnamon and clove was obtained.

$$
\begin{aligned}
& \mathrm{Y} 12=54.20199-19.06672 \mathrm{X} 1-20.65111 \mathrm{X} 2- \\
& 3.21673 \mathrm{X} 12-1.80286 \mathrm{X} 22+14.75000 \mathrm{X} 1 \mathrm{X} 2
\end{aligned}
$$

According to the interaction equation, the interaction curves of cinnamon and clove are obtained, as shown in Figure 3.

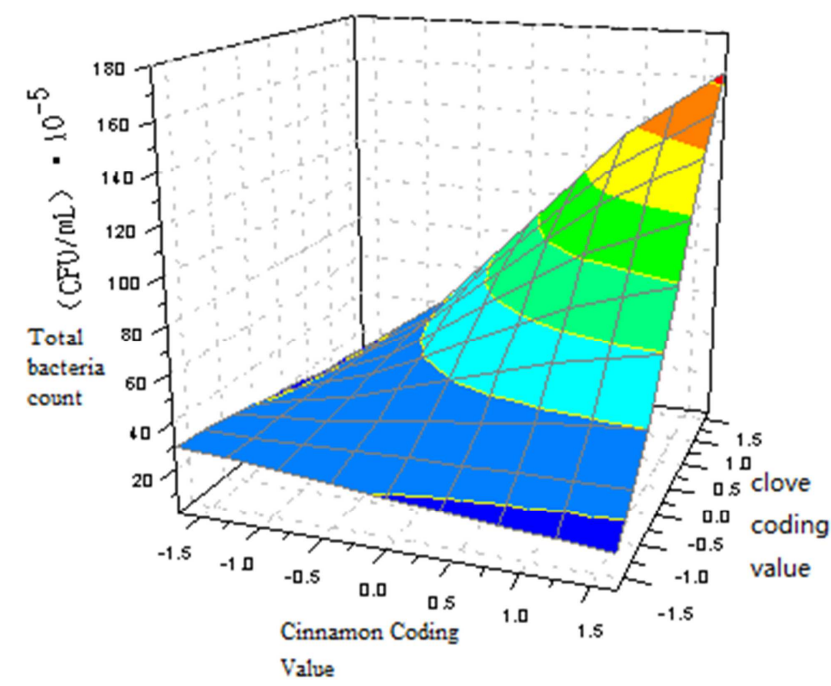

Figure 3. The curve of interaction between Cinnamomum cassia and Syzygiumaromaticum

Figure 3 showd that when cinnamon and clove were added the least, the total bacterial count reached the minimum, and the bacteriostasis effect of compound preservatives was the best.

\subsection{Determination of the Optimum Concentration of Composite Preservative}

By analyzing the results of three-factor quadratic general rotary design test, it could be concluded that the total number of bacteria $(\mathrm{CFU} / \mathrm{mL}) / 10^{5}$ reached the lowest level. When $\mathrm{Y}=$ $7.8^{*} 10^{5}(\mathrm{CFU} / \mathrm{mL})$, the coding values of the three preservatives were as follows: $\mathrm{X}_{1}=-1.682, \mathrm{X}_{2}=1.682$, $\mathrm{X}_{3}=1.000$

Converting the coding value to the actual value, the optimum concentration of three preservatives could be obtained as follows: Cinnamon $0.063 \mathrm{~g} / \mathrm{mL}$; Clove 1.000 $\mathrm{g} / \mathrm{mL}$; Star anise $0.810 \mathrm{~g} / \mathrm{mL}$.

\subsection{Sensory Evaluation Results}

Compared with No.1 sample without compound preservatives, the sample with compound preservatives had no nausea and nausea taste, and has a light flavoring taste, so that the meat belly did not taste greasy.

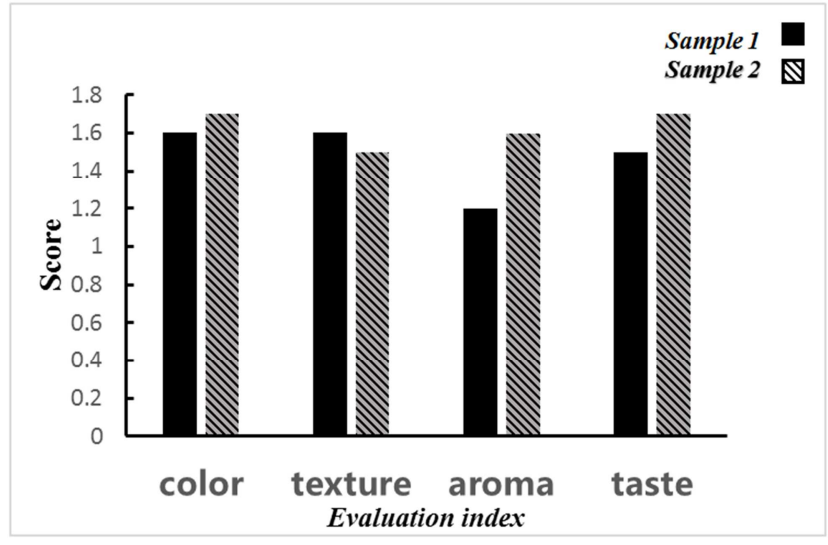

Figure 4. Sensory evaluation.

\subsection{Determination of Microbial Indicators During Storage}

Table 4. The results of microbial index determination on sample (1).

\begin{tabular}{llll}
\hline & Day 0-6 & Day 7 & Day 8 \\
\hline total numbers of colony (CFU/g) & $<10$ & 62000 & 75000 \\
Coliform population (MPN/100g) & 0 & 0 & 130 \\
\hline
\end{tabular}

Table 5. The results of microbial index determination on sample (2).

\begin{tabular}{lllll}
\hline & Day 0-8 & Day 9 & Day 10 & Day 11 \\
\hline total numbers of colony (CFU/g) & $<10$ & 7800 & 55000 & 98000 \\
Coliform population (MPN/100g)) & 0 & 0 & 300 & 330 \\
\hline
\end{tabular}

On the 7th and 8th days of storage, the total number of colonies and coliforms exceeded the national standards, respectively. The shelf life of No. 1 sample was $9 \mathrm{~d}$. On the 10th day of storage, the total number of colony and coliform bacteria exceeded the requirements of the national standard, so the shelf life of No. 2 pork belly sample was 9 days.

\section{Conclusion}

In this experiment, the optimum formula of compositive preservatives was obtained by three-factor quadratic general rotary design method: cinnamon $0.063 \mathrm{~g} / \mathrm{mL}$, clove 1.000 $\mathrm{g} / \mathrm{mL}$, anise $0.810 \mathrm{~g} / \mathrm{mL}$. It can extend the shelf life for 9 days when applied to Beijing sausages.

\section{Acknowledgements}

We thank France the Teaching Group of Food Chemistry, Faculty of Food Science and Engineering of Beijing University of Agriculture for laboratory assistance. Molecular analyses were supported by Beijing Key Laboratory of Agricultural, Product Detection and Control of Spoilage Organisms and Pesticide Residue. We thank Beijing Innovation Consortium of Swine Researeh System and Graduation Design of the Practical Training Program for the Cross-Cultivation of High-Level Talents in Beijing Colleges and Universities for support. 


\section{References}

[1] Wu Xiaoli, Zhang Xiangsheng, Jiang Aimin, etc. Research on fresh-keeping Technology of Meat products with Sauce and Sauce [J]. Meat Industry, 2014 (7): 46-50.

[2] Wang Tingting, Li Hongjun. The role of extract of spices in Meat Preservation and its response With [J]. Food Industry Science and Technology, 2010, 31 (2): 359-361.

[3] Xu Yan, Huang Jinghua. Extraction method of natural preservative from rosemary and its bacteriostatic effect [J]. Amino acids and Biological Resources, 2007, 29 (2): 1-4.

[4] Li Lei, Cui Liwei, Zhang Ran, Hao Xiuzhen and Wang Mingqi. Application of three spice essential oils in the preservation of steak film [J]. Food Science and Technology, 2019 (02): $138-142+151$.

[5] Meng Jun, Peng Xiuli, Zhang Junsong and Luo Liqiang. Study on the bacteriostasis and volatile components of three spices extracts [J]. Chinese condiments. 2019, 44 (01): 40-44.

[6] Ai Youwei, Wang Limei and Yan Hushan. Study on the antimicrobial activity of common spices extracts [J]. Food industry. 2018, 39 (09): 167-170.

[7] Ai Youwei, Hou Wenfu, Zhang Jie and Wang Hongxun. Study on the change rule of main spices in the process of marinating soy sauce and brine products [J]. Food industry. 2018, 39 (08): 191-194.

[8] Zheng Haiyun. Study on the effect of spice extracts on the quality of Chaoshan beef balls [D]. South China University of Technology, 2018.

[9] Xie Qiang, Miao Shuping, Shi Shouji, Chen Jinbiao and Xia Xiuhua. Different effects of six spice extracts on food-borne pathogens and probiotics [J]. Food Research and Development, 2018, 39 (04): 38-42.

[10] Wang Pengxiang. Research status of bacteriostasis and antioxidant activity of edible spices [J]. Modern Food, 2018 (01): 21-23.

[11] Chen Maoquan, Ying Junhui, Wang Dongming. Shadows of several spices on the quality of low-salt pickles Sound [J]. Food Science and Technology, 2014 (3): 261-263.

[12] Bayoub K. Antibacterial activities of the crude ethanol extracts of medicinal plants against Listeria monocytogenes and some other pathogenic strains [J]. 2010, 9 (27): 4251-4258.

[13] Ma Lizhen. Study on Comprehensive Biological and physical Preservation techniques and Preservation effects of chilled Pork Research [D]. Beijing: China Agricultural University, 2003.

[14] Benmalek Y, Yahia O A, Belkebir A, et al. Anti-microbial and anti-oxidant activities of Illicium verum, Crataegus oxyacantha ssp monogyna and Allium cepa red and white varieties [J]. Bioengineered, 2013, 4 (4): 244-248.

[15] Wang Tongyu, Tian Yuhong and Zhou Xiaoliu. Study on the antibacterial activity of the water-soluble volatile components of the eight-angle-type fragrant incense [J]. Chinese seasoning, 2010 (6): 29-32.

[16] Tian Feng, Li Xiao, Cui Yuqian, Xu Lingyi, QiJinHua. Study on bacteriostatic effect of flavoring extract on Pseudomonas, staphylococci and pyracyclomycetes [J]. Journal of Beijing Agricultural College, 2017, 32 (02): 10-14.

[17] Zhu Jiankai. Application of spices and protease in tenderization and preservation of beef [J]. Meat Industry, 2008 (5): 33-35.

[18] Li Lanxiao, Du Jinhua, Shang Riling, et al. Study on Optimization of cellulase conditions for solid-state fermentation of Aspergillus Niger by quadratic general rotary test design [J]. Food and Fermentation Industry, 2008 (1): 84-87.

[19] Li Yongqiang, Yang Shihua, Bi Xiaofei, et al. Study on the optimization of the extraction process of red pigment from Trigonella praecox by twice general rotary test design $[\mathrm{J}]$. China Food Additive, 2010 (5): 68-72. 